\title{
Agronomic performance of 'BRS Kampai' peach on 15 clonal rootstocks and own-rooted trees in Pelotas-RS, Brazil
}

\author{
Newton Alex Mayer ${ }^{1}$, Bernardo Ueno ${ }^{1}$, Luis Eduardo Correa Antunes ${ }^{1}$, \\ Gilberto Nava ${ }^{1}$, Fernanda Maisa Roth ${ }^{2}$
}

\begin{abstract}
The clonal rootstocks allows preservation of mother tree genetic characteristics, such as vigor and produce a fasciculated root system, with an abundant thin roots. The objective of this research was to determine the effect of fifteen clonal genotypes as rootstocks on the agronomic performance of 'BRS Kampai' peach, as well as the technical feasibility of own-rooted trees (without rootstock) in a no-irrigated field condictions, in Pelotas-RS, Brazil. Bagged nursery trees were planted at the field in August, 2014, under a randomized block design with four replications of one tree per plot, and evaluations of trunk diameter, trunk cross-sectional area (TCSA), yield efficiency, annual and accumulated fruit yield per tree and per hectare were carried out until 2019. In the adopted experimental conditions, we conclude that 'Barrier' $(P$. persica $\mathrm{x} P$. davidiana), 'Cadaman' ( $P$. persica $\times$ P. davidiana), G x N.9 (P. persica $\times$ P. dulcis), 'Ishtara' [( $P$. cerasifera $\mathrm{x}$ $P$. salicina $) \times(P$. cerasifera $\times$ P. persica $)]$ and 'Santa Rosa' $(P$. salicina $)$ do not have potential as a rootstock of 'BRS Kampai' peach due irregular bud breaking of scion, small fruits and/or low fruit yield. Clone 15 (P. mume) stands out for increasing fruit weight and maintaining satisfactory fruit yield per tree. 'Flordaguard' $(P$. persica) is the best rootstock tested, which is vigorous, do not present any undesirable agronomic characteristics and induce the highest yields per tree and per hectare, exceeding $50 \mathrm{t} \mathrm{ha}^{-1}$ in accumulated yield. Own-rooted trees of 'BRS-Kampai' peach have technical feasibility, as long as pruning is properly carried out.
\end{abstract}

Index terms: Prunus spp., vegetative propagation, softwood cutting, interspecific budding.

\section{Performance agronômica do pessegueiro 'BRS Kampai' sobre 15 porta- enxertos clonais e de plantas autoenraizadas em Pelotas-RS, Brasil}

\section{Corresponding author: alex.mayer@embrapa.br \\ Received: September 08, 2020 Accepted: January 18, 2021}

Copyright: All the contents of this journal, except where otherwise noted, is licensed under a Creative Commons Attribution License.

\section{(cc) $\mathrm{EY}$}

Resumo - Os porta-enxertos clonais permitem a preservação das características genéticas da plantamatriz, tais como o vigor, e produzem um sistema radicular fasciculado, com abundante quantidade de radicelas. O objetivo do presente trabalho foi determinar o efeito de quinze porta-enxertos clonais na performance agronômica de pessegueiros 'BRS Kampai', bem como a viabilidade técnica do uso de plantas autoenraizadas (sem porta-enxerto) em condição de campo sem irrigação, em Pelotas-RS, Brasil. Mudas produzidas em sacos plásticos foram plantadas no campo, em agosto de 2014, sob delineamento de blocos casualizados, com quatro repetições, de uma planta por parcela, e avaliações de diâmetro do tronco, área da secção transversal do tronco, eficiência produtiva, produção de frutos por planta e por hectare anual e acumulada foram realizadas até 2019. Nas condições experimentais adotadas, conclui-se que 'Barrier' (P. persica x P. davidiana), 'Cadaman' (P. persica $\times$ P. davidiana), G x N.9 (P. persica $\times$ P. dulcis), 'Ishtara' [(P. cerasifera $\times$ P. salicina) $\mathrm{x}(P$. cerasifera $\mathrm{x} P$. persica $)$ e 'Santa Rosa' $(P$. salicina $)$ não apresentam potencial como portaenxerto do pessegueiro 'BRS Kampai' devido à irregular quebra de dormência das gemas da copa, frutos pequenos e/ou baixa produção. O Clone 15 (P. mume) destaca-se por aumentar o peso do fruto, mantendo satisfatória produção por planta. O 'Flordaguard' ( $P$. persica) é o melhor portaenxerto testado, que é vigoroso, não apresenta nenhuma característica agronômica indesejável e induz as maiores produções por planta e por hectare, superando $50 \mathrm{t} \mathrm{ha}^{-1}$ na produção acumulada. Pessegueiros autoenraizados de 'BRS-Kampai' apresentam viabilidade técnica de cultivo, desde que as podas sejam adequadamente realizadas.

Termos para indexação: Prunus spp., propagação vegetativa, estaquia herbácea, enxertia interespecífica.

\footnotetext{
${ }^{1}$ Agricultural Engineer, Dr., Researcher at Embrapa Clima Temperado. Pelotas-RS, Brazil. E-mail: alex.mayer@embrapa.br (ORCID: 00000001-6689-8202); bernardo.ueno@embrapa.br(ORCID: 0000-0002-0355-6907); luis.antunes@embrapa.br (ORCID: 0000-0002-0341-1476); gilberto.nava@ embrapa.br(ORCID: 0000-0002-8059-1052)

${ }^{2}$ Master student of Universidade Federal de Pelotas, Capes scholarship. E-mail:fernanda.ufpel@hotmail.com ${ }^{\text {(ORCID: 0000-0002-6140-4418) }}$
} 


\section{Introduction}

In grafted fruit trees, rootstock is a part of the tree whose purpose is to receive scion bud during nursery tree production. With this successful union, tree will be composed of two distinct genotypes that will coexist for entire life with the advantage that original genetic identity of each genotype remains preserved (DAVIES Jr., 2017). Since it is a root system of the grafted trees, rootstock must be adapted to live with physical, chemical and biological soil conditions, soil temperature and moisture variations, which are specific characteristics of each fruit producing area. Therefore, for rootstock recommendation purposes, local research should be done and considering climate and soil conditions diversity (LAYNE, 1987; REIGHARD; LORETI, 2008; MAYER et al., 2014a).

In the Rio Grande do Sul State, the largest Brazilian peach producer (AGRIANUAL, 2020), the scion cultivars Aldrighi and Capdeboscq, released for processing purposes, were also widely used in the past as rootstocks, due to seed availability in the processing industries, satisfactory seed germination and adaptation to the Pelotas peach growing area (FINARDI, 1998; MAYER et al., 2014a). However 'Capdeboscq' and 'Aldrighi' are susceptible to the nematode Meloidogyne incognita (MAUCH et al., 1991); 'Capdeboscq' is susceptible to M. ethiopica (SOMAVILLA, 2008), to the ring nematode Mesocriconema xenoplax (CARNEIRO et al., 1998) and to soil waterlogging (GUERRA et al., 1992). With the development of new peach cultivars for processing in Brazil (RASEIRA et al., 2014), 'Aldrighi' and 'Capdeboscq' were no longer planted since the 1980s, so its seed availability is now restricted to rare nurseries that keep mother trees for seed production (MAYER et al., 2014a). Nurseries in Southern Brazil that do not have their own rootstock mother trees blocks, make use of seed mixture from different scion peach cultivars obtained in the canning industry (residue from peach canning process), which increases genetic variability of rootstocks, prevents their standardization and reduces the useful orchards life (MAYER; UENO, 2012; MAYER et al., 2014a; MAYER et al., 2017).

Due to low yield of peach orchards in Rio Grande do Sul (11.1 tha $\mathrm{t}^{-1}$ ) (AGRIANUAL, 2020), problems with the Peach Tree Short Life syndrome (PTSL) and tradition of obtaining seed mixtures from canning industry to produce rootstocks (MAYER; UENO, 2012; MAYER et al., 2014a), new rootstock assessments in this state started to be developed in the last 20 years, however with some divergent results due to edaphoclimatic differences and scion/rootstock interactions (DE ROSSI et al., 2004; ROCHA et al., 2007; PICOLOTTO et al., 2009; GALARÇA et al., 2013; BARRETO et al., 2017; MAYER et al., 2019). In these studies, the genetic variability tested as a rootstock was quite limited, restricted to one selection
("Viamão") and eight cultivars of P. persica (Capdeboscq, Aldrighi, Tsukuba-1, Nemaguard, Flordaguard, Okinawa, GF305 and Pavia Moscatel), one interspecific hybrid ('Hamsen 2168') and japanese apricot (P. mume), all seed propagated, which does not guarantee genetic fidelity of mother tree and preservation of its characteristics of interest, due to genetic segregation and cross-pollination (MILLER et al., 1989; BECKMAN, 1998).

Other Prunus species or interspecific hybrids have been studied and commercially used as rootstock for peach in several countries, being especially indicated for calcareous soils and/or replanting areas due to the lower toxicity of root exudates and biochemical inhibitors than those produced by $P$. persica. However, graft incompatibility is a limiting factor and interspecific crossings or assessments for each edaphoclimatic condition and scion/rootstock combination should be performed (BUSSI et al., 2002; REIGHARD; LORETI, 2008; DUVAL, 2015). Prunus mume has potential for use as a rootstock for peaches and nectarines (CAMPO DALL'ORTO et al., 1992), however, if propagated by seeds, genetic variability is very accentuated, which requires selection and vegetative propagation of the best clones (PEREIRA et al., 2007; MATHIAS et al., 2008; MAYER et al., 2015). Soil watterlogging is one of the main winter problems in Rio Grande do Sul (FINARDI, 1995), being plum trees more tolerant (GUERRA et al., 1992; DUVAL, 2015). However, they usually have graft incompatibility problems, if budded with peach (NEVES et al., 2017). 'Cadaman', 'GF 677', 'Penta', 'MrS 2/5', 'Julior', 'Nemaguard' and 'INIA Tsukuba ${ }^{\circ} 1$ ' rootstocks has been successfully tested in Uruguay for peaches (CABRERA; RODRIGUEZ, 2014).

On the other hand, a common question is whether there is a need for rootstock when it does not have specific characteristics suitable for that edaphoclimatic condition and/or does not add any advantageous characteristics to the scion. Peach and nectarine scions can be easily propagated by softwood cuttings under intermittent mist system, eliminating the use of rootstock and reducing time for nursery tree production (COUVILLON, 1985; MAYER et al., 2017), with satisfactory field development of some cultivars (SANTANA et al., 2020; JIMENEZ et al., 2020). The objective of this research was to determine the effect of fifteen clonal genotypes as rootstocks on the agronomic performance of 'BRS Kampai' peach, as well as the technical feasibility of own-rooted trees (without rootstock) in a no-irrigated field condictions, in PelotasRS, Brazil. 


\section{Material and Methods}

Fifteen genotypes (selections, cultivars, species or interspecific hybrids) were propagated vegetatively by softwood cuttings from November, 2012 to February, 2013 , to be tested as rootstocks for 'BRS Kampai' peach. The identification, main features and references can be found in Table 1. Sofwood shoots were collected from mother trees of the "Prunus rootstock collection" of Embrapa Clima Temperado (Pelotas-RS), managed for this purpose, with drastic winter pruning to stimulate new and intense sprouting. Softwood cuttings with $15 \mathrm{~cm}$-long were prepared, keeping three distal nodes with all their leaves cut in half to reduce leaf transpiration. The cutting base $(3 \mathrm{~cm})$ was treated with indolbutyric acid at 3,000 mg. $L^{-1}$ and placed in fine vermiculite for rooting under intermittent mist system inside a greenhouse (MAYER et al., 2013).

After rooting period, the rooted cuttings classified as suitable were transplanted into perforated plastic bags $(30 \mathrm{~cm} \times 18 \mathrm{~cm})$ containing commercial substrate based on pine bark and peat, and conducted on a single stem. In the subsequent Summer (January, 2014), rootstocks were budded by "T-inverted" method with 'BRS Kampai' peach [Prunus persica (L.) Batsch] (RASEIRA et al., 2010). Own-rooted nursery trees (without rootstock) of 'BRS Kampai' were also produced by softwood cuttings to be tested (MAYER et al., 2013).

The experiment was installed at no-irrigated field conditions, altitude of 45-48 m.a.s.1. at Embrapa Clima Temperado ( $9^{\text {th }}$ district of Pelotas, Rio Grande do Sul State, Brazil). Pre-planting soil samples showed, in general, satisfactory $\mathrm{pH}$ and nutrient levels, except for very low or low values of $\mathrm{P}, \mathrm{K}$ and $\mathrm{Mn}$, which were corrected with pre-planting and growing fertilization. Soil organic matter contents were also low $(<1.3 \%)$ and remained stable throughout the experiment (CQFS, 2004; CQFS, 2016). Bagged nursery trees were planted on August, $21^{\text {th }}, 2014$, with $6.0 \times 3.0 \mathrm{~m}$ spacing $\left(555\right.$ trees $\left.\mathrm{ha}^{-1}\right)$ and conducted in "open vase" training system, with 5 to 7 main scaffolds (PEREIRA; RASEIRA, 2014a). Hand fruit thinning was carried out using the criterion of minimum distance between fruits, being 8 to $10 \mathrm{~cm}$ for vigorous shoots, and 12 to $15 \mathrm{~cm}$ for short shoots (PEREIRA; RASEIRA, 2014b). A randomized block design was adopted, with four replications of one tree per plot, with treatments consisting of fifteen clonal rootstocks and the own-rooted scion trees, totaling 16 treatments (Table 1) and 64 plots.

Rainfall distribution was uneven throughout the months, however, in the six years of this trial, annual rainfall volumes were greater than the annual historical average. Chilling hours accumulation was below than historical average in all six years, but it was even lower in $2015\left(87\right.$ hours $\left.\leq 7.2^{\circ} \mathrm{C}\right)$ and $2017\left(77\right.$ hours $\left.\leq 7.2^{\circ} \mathrm{C}\right)$ (AGROMET, 2020), when accumulated chilling hours did not reach a half of the 'BRS Kampai' necessity, which is estimated at 200 hours per year (RASEIRA et al., 2010).

Trunk diameter (TD) evaluations were performed at planting and annually during their Winter (July), with a digital caliper, taking measurement $5 \mathrm{~cm}$ above the grafting point. In own-rooted trees, evaluation was made 5 $\mathrm{cm}$ above the insertion of trunk on original cutting. Trunk cross-sectional area (TCSA) was calculated, as follows: TCSA $=\pi \times \mathrm{R}^{2}$, where TCSA is expressed in $\mathrm{cm}^{2} ; \pi=$ 3.1416; $\mathrm{R}=$ radius, in $\mathrm{cm}$. The following variables were evaluated along four years (2016, 2017, 2018 and 2019): a) fruit number per tree (FNT), determined annually some days before fruit ripening (at the end of October or beginning of November), by counting all fruits per tree. The accumulated fruit number per tree (four crops) were also calculated; b) fruit weight (FW), at harvest time, a sample of fruits per plot was collected and weighed on a digital scale, determining average fruit weight $(\mathrm{g})$ for each year and average for the four crops; c) fruit yield per tree (FYT), calculated by fruit number per tree and fruit weight, and expressed as $\mathrm{kg}$ tree $\mathrm{e}^{-1} ; \mathrm{d}$ ) accumulated fruit yield per tree (AFYT), determined by the sum of four years of yield per tree and expressed as $\mathrm{kg}$ tree $^{-1} ; \mathrm{e}$ ) yield efficiency (YE), determined by $\mathrm{YE}=\mathrm{FYT} / \mathrm{TCSA}$, expressed as $\mathrm{kg} \mathrm{cm}^{2} ; \mathrm{f}$ ) fruit yield per hectare $(\mathrm{FYH})$ : determined by $\mathrm{FYH}=(\mathrm{FYT}$ $\mathrm{x} \mathrm{n}^{\circ}$ of trees per hectare)/1000, and expressed in $\mathrm{t} \mathrm{ha}^{-1} ; \mathrm{g}$ ) accumulated fruit yield per hectare (AFYH) determined by the sum of four years of yield per hectare and expressed as t ha ${ }^{-1}$. The results were subjected to analysis of variance by the F test and the means compared by the Scott-Knott test, using the SASM-Agri software (CANTERI et al., 2001). 
Table 1. Fifteen Prunus spp. genotypes as clonal rootstocks for 'BRS Kampai' peach, and own-rooted scion trees, with main features and references. Embrapa Clima Temperado, Pelotas-RS, Brazil.

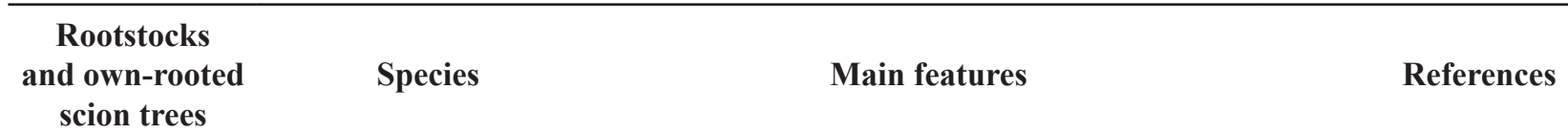

Barrier $\quad$ P.persica $\mathrm{x}$ P. davidiana

Waterlogging tolerance; drought tolerance better Reighard (2002); Reighard and than peach seedlings.

Loreti (2008).

Cadaman P.persica $\times$ P. davidiana

Waterlogging tolerance; resistant to Meloidogyne incognita, $M$. arenaria and $M$. hispanica; drought tolerance better than peach seedlings; suitable for replanting areas.

Di Vito et al. (2002); Bussi et al. (2002); Reighard (2002); Reighard and Loreti (2008).

Resistant to $M$. javanica and $M$. incognita race 2 ; red leaf.

Rossi et al. (2002).

\begin{tabular}{lll} 
Gx N.9 P.persica x P. dulcis & $\begin{array}{l}\text { Resistant to M. javanica and M. incognita race Rossi et al. (2002). } \\
\text { 2; red leaf. }\end{array}$ \\
\hline
\end{tabular}

$\begin{array}{lll}\text { Capdeboscq } & \text { P. persica } & \begin{array}{l}\text { Adaptation to climate and soil conditions of Rio Finardi (1998). } \\ \text { Grande do Sul State. }\end{array}\end{array}$

Easy propagation by softwood cuttings; resistance

Rigitano P. mume to M. javanica and M. incognita; less vigor than Mayer et al. (2006); Pereira et 'Okinawa'; induces greater fruit weight, size and al., 2007; Mathias et al. (2008). soluble solids for 'Aurora-1' peach.

\section{Easy propagation by softwood cuttings; resistance}

Clone $15 \quad$ P. mume to M. javanica and M. incognita; induces greater Mayer et al. (2006); Mathias et fruit weight, size and soluble solids for 'Aurora-1' al. (2008). peach.

I-67-52-4 P. persica Increase genetic variability of the species. $\quad$ No information.

Tsukuba-1 P. persica

Tsukuba-2 P. persica

Tsukuba-3 P. persica

Okinawa $\quad$ P. persica

\begin{tabular}{ll} 
Flordaguard & $\begin{array}{l}\text { P.persica }\left(6^{\text {th }} \text { generation of }\right. \\
\left.\text { 'Chico } 11^{\prime} \times \text {. davidiana }\right)\end{array}$ \\
\hline Nemared & P. persica \\
\hline Ishtara & $(P$. cerasifera $\times$ P. salicina $)$ \\
& $\begin{array}{l}\text { X }(P . \text { cerasifera } \times \\
\text { persica })\end{array}$ \\
\hline
\end{tabular}

Santa Rosa

P. salicina

Waterlogging tolerance.
Waterlogging tolerance; resistance to M. incognita Reighard (2002); Rossi et al. race 2 and $M$. javanica; red leaf.
(2002).
Waterlogging tolerance; resistance to M. incognita Reighard (2002); Rossi et al. race 2 and $M$. javanica; red leaf.
(2002).

Waterlogging tolerance; resistance to $M$. incognita
race 2 and $M$. javanica; red leaf.

Reighard (2002); Rossi et al. (2002).

Easy propagation by softwood cuttings; freestone Nachtigal (1999); Rossi et al. and good seed germination; low chill requirement; (2002); Mayer et al. (2003); resistance to $M$. incognita and M. javanica; Mayer et al. (2005); Ferguson tolerant to M. floridensis. and Chaparro (2008).

Resistant to M. javanica, M. floridensis and $M$. incognita races 1 and 3; low chill requirement; freestone and good seed germination; red leaf; high vigor.

Sherman et al. (1991); Ferguson and Chaparro (2008); Duval (2015).

Resistant to root-knot nematodes; red leaf; Ramming and Tanner (1983); vigorous growth; good anchorage.

Layne (1987).

Resistant to M. incognita, M. javanica, $M$. arenaria, M. hapla and $M$. hispanica; less vigor than GF 677; waterlogging tolerance; resistant to Armillaria mellea.

Di Vito et al. (2002); Loreti and Massai (2002); Beckman and Lang (2003); Reighard (2002).

Guerra et al. (1992).

High vigor; easy propagation by softwood cuttings; to check the technical feasibility of nursery trees without rootstock.
Mayer et al. (2013); Neves et al. (2017).
Own-rooted 'BRS
Kampai' persica

nes without rootstock. 


\section{Results and Discussion}

The bagged nursery trees were homogeneous at planting, with no differences in trunk diameter and TCSA. Over the years, differences in these two variables have been detected (exception in 2018), being normally greater on Clone 15 and 'Flordaguard' (Tables 2 and 3). At the end of this study, trees on Clone 15, 'Tsukuba-1', 'Okinawa' and 'Flordaguard', as well as own-rooted trees, were significantly more vigorous (Table 3 ).

Table 2. Effects of clonal rootstocks used for 'BRS Kampai' peach and own-rooted trees (without rootstock) in trunk diameter (mm). Embrapa Clima Temperado, Pelotas-RS, Brazil.

\begin{tabular}{lcccccc}
\hline \multirow{2}{*}{ Rootstock } & \multicolumn{5}{c}{ Trunck diameter $(\mathrm{mm})$} \\
\cline { 2 - 7 } & 2014 & 2015 & 2016 & 2017 & 2018 & 2019 \\
\hline Barrier & $4.96 \mathrm{a}$ & $32.20 \mathrm{~b}$ & $63.66 \mathrm{c}$ & $79.42 \mathrm{~b}$ & $96.79 \mathrm{a}$ & $104.07 \mathrm{~b}$ \\
\hline Cadaman & $4.57 \mathrm{a}$ & $36.26 \mathrm{a}$ & $70.89 \mathrm{c}$ & $93.61 \mathrm{~b}$ & $100.94 \mathrm{a}$ & $108.71 \mathrm{~b}$ \\
\hline GxN.9 & $4.40 \mathrm{a}$ & $34.93 \mathrm{a}$ & $70.34 \mathrm{c}$ & $92.72 \mathrm{~b}$ & $107.56 \mathrm{a}$ & $110.54 \mathrm{~b}$ \\
\hline Capdeboscq & $4.88 \mathrm{a}$ & $35.46 \mathrm{a}$ & $77.94 \mathrm{~b}$ & $99.81 \mathrm{a}$ & $118.26 \mathrm{a}$ & $123.23 \mathrm{a}$ \\
\hline Rigitano & $4.60 \mathrm{a}$ & $34.40 \mathrm{a}$ & $76.02 \mathrm{~b}$ & $84.61 \mathrm{~b}$ & $99.75 \mathrm{a}$ & $116.74 \mathrm{~b}$ \\
\hline Clone 15 & $5.13 \mathrm{a}$ & $40.32 \mathrm{a}$ & $97.06 \mathrm{a}$ & $110.24 \mathrm{a}$ & $124.66 \mathrm{a}$ & $130.89 \mathrm{a}$ \\
\hline I-67-52-4 & $4.29 \mathrm{a}$ & $32.29 \mathrm{~b}$ & $63.47 \mathrm{c}$ & $83.80 \mathrm{~b}$ & $94.41 \mathrm{a}$ & $113.11 \mathrm{~b}$ \\
\hline Tsukuba-1 & $4.88 \mathrm{a}$ & $32.97 \mathrm{~b}$ & $70.50 \mathrm{c}$ & $90.18 \mathrm{~b}$ & $109.40 \mathrm{a}$ & $130.03 \mathrm{a}$ \\
\hline Tsukuba-2 & $4.37 \mathrm{a}$ & $26.32 \mathrm{~b}$ & $64.47 \mathrm{c}$ & $78.66 \mathrm{~b}$ & $92.73 \mathrm{a}$ & $104.61 \mathrm{~b}$ \\
\hline Tsukuba-3 & $4.49 \mathrm{a}$ & $26.89 \mathrm{~b}$ & $64.35 \mathrm{c}$ & $87.28 \mathrm{~b}$ & $110.62 \mathrm{a}$ & $117.80 \mathrm{~b}$ \\
\hline Okinawa & $4.36 \mathrm{a}$ & $31.03 \mathrm{~b}$ & $74.51 \mathrm{c}$ & $93.08 \mathrm{~b}$ & $107.77 \mathrm{a}$ & $125.38 \mathrm{a}$ \\
\hline Flordaguard & $5.24 \mathrm{a}$ & $42.33 \mathrm{a}$ & $88.13 \mathrm{a}$ & $107.65 \mathrm{a}$ & $113.95 \mathrm{a}$ & $153.00 \mathrm{a}$ \\
\hline Nemared & $3.45 \mathrm{a}$ & $32.53 \mathrm{~b}$ & $70.87 \mathrm{c}$ & $101.00 \mathrm{a}$ & $107.28 \mathrm{a}$ & $121.34 \mathrm{a}$ \\
\hline Ishtara & $4.39 \mathrm{a}$ & $25.56 \mathrm{~b}$ & $52.57 \mathrm{c}$ & $66.64 \mathrm{~b}$ & $81.96 \mathrm{a}$ & $91.28 \mathrm{~b}$ \\
\hline Santa Rosa & $4.42 \mathrm{a}$ & $28.50 \mathrm{~b}$ & $61.33 \mathrm{c}$ & $85.06 \mathrm{~b}$ & $97.09 \mathrm{a}$ & $109.05 \mathrm{~b}$ \\
\hline Own-rooted 'BRS Kampai' & $5.33 \mathrm{a}$ & $36.52 \mathrm{a}$ & $82.39 \mathrm{~b}$ & $116.89 \mathrm{a}$ & $129.24 \mathrm{a}$ & $142.07 \mathrm{a}$ \\
\hline F & $1.6654^{\text {ns }}$ & $3.7422^{* *}$ & $5.2501^{* *}$ & $2.7810^{* *}$ & $1.8095^{\text {ns }}$ & $2.4718^{* *}$ \\
\hline Footstock & $0.4745^{\text {ns }}$ & $2.0166^{\text {ns }}$ & $8.7211^{* *}$ & $5.8475^{* *}$ & $4.9982^{* *}$ & $8.4060^{* *}$ \\
\hline CV (\%) & 15.44 & 14.86 & 13.36 & 16.91 & 17.25 & 16.42 \\
\hline
\end{tabular}

Means followed by different letters in the column differ from each other by Scott-knott test. ${ }^{* *}$ significant at $99 \%$ confidence; ${ }^{\text {ns }}$ not significant.

Table 3. Effects of clonal rootstocks used for 'BRS Kampai' peach and own-rooted trees (without rootstock) in trunk cross-sectional area $\left(\mathrm{cm}^{2}\right)$. Embrapa Clima Temperado, Pelotas-RS, Brazil.

\begin{tabular}{lcccccc}
\hline \multirow{2}{*}{ Rootstock } & \multicolumn{5}{c}{ Trunk cross-sectional area $\left(\mathrm{cm}^{2}\right)$} \\
\cline { 2 - 7 } & 2014 & 2015 & 2016 & 2017 & 2018 & 2019 \\
\hline Barrier & $0.20 \mathrm{a}$ & $8.65 \mathrm{c}$ & $33.56 \mathrm{c}$ & $53.19 \mathrm{~b}$ & $76.35 \mathrm{a}$ & $87.71 \mathrm{~b}$ \\
\hline Cadaman & $0.17 \mathrm{a}$ & $10.34 \mathrm{~b}$ & $39.88 \mathrm{c}$ & $70.41 \mathrm{~b}$ & $83.31 \mathrm{a}$ & $93.96 \mathrm{~b}$ \\
\hline GxN.9 & $0.15 \mathrm{a}$ & $9.83 \mathrm{~b}$ & $40.86 \mathrm{c}$ & $72.70 \mathrm{~b}$ & $92.36 \mathrm{a}$ & $100.69 \mathrm{~b}$ \\
\hline Capdeboscq & $0.19 \mathrm{a}$ & $9.97 \mathrm{~b}$ & $49.13 \mathrm{c}$ & $80.78 \mathrm{a}$ & $114.23 \mathrm{a}$ & $122.75 \mathrm{~b}$ \\
\hline Rigitano & $0.17 \mathrm{a}$ & $9.42 \mathrm{~b}$ & $45.58 \mathrm{c}$ & $58.33 \mathrm{~b}$ & $81.40 \mathrm{a}$ & $107.89 \mathrm{~b}$ \\
\hline Clone 15 & $0.21 \mathrm{a}$ & $13.13 \mathrm{a}$ & $75.52 \mathrm{a}$ & $98.92 \mathrm{a}$ & $125.32 \mathrm{a}$ & $141.91 \mathrm{a}$ \\
\hline I-67-52-4 & $0.15 \mathrm{a}$ & $8.22 \mathrm{c}$ & $32.11 \mathrm{c}$ & $56.15 \mathrm{~b}$ & $72.57 \mathrm{a}$ & $108.06 \mathrm{~b}$ \\
\hline Tsukuba-1 & $0.19 \mathrm{a}$ & $8.58 \mathrm{c}$ & $39.39 \mathrm{c}$ & $64.11 \mathrm{~b}$ & $99.00 \mathrm{a}$ & $134.40 \mathrm{a}$ \\
\hline Tsukuba-2 & $0.16 \mathrm{a}$ & $5.48 \mathrm{c}$ & $32.92 \mathrm{c}$ & $49.50 \mathrm{~b}$ & $67.81 \mathrm{a}$ & $87.49 \mathrm{~b}$ \\
\hline Tsukuba-3 & $0.16 \mathrm{a}$ & $5.76 \mathrm{c}$ & $33.49 \mathrm{c}$ & $61.26 \mathrm{~b}$ & $99.60 \mathrm{a}$ & $113.80 \mathrm{~b}$ \\
\hline Okinawa & $0.16 \mathrm{a}$ & $7.76 \mathrm{c}$ & $45.25 \mathrm{c}$ & $70.44 \mathrm{~b}$ & $95.42 \mathrm{a}$ & $129.07 \mathrm{a}$ \\
\hline Flordaguard & $0.22 \mathrm{a}$ & $14.35 \mathrm{a}$ & $61.68 \mathrm{~b}$ & $92.79 \mathrm{a}$ & $103.79 \mathrm{a}$ & $187.76 \mathrm{a}$ \\
\hline Nemared & $0.09 \mathrm{a}$ & $8.50 \mathrm{c}$ & $40.28 \mathrm{c}$ & $81.24 \mathrm{a}$ & $90.64 \mathrm{a}$ & $119.35 \mathrm{~b}$ \\
\hline Ishtara & $0.16 \mathrm{a}$ & $5.22 \mathrm{c}$ & $21.71 \mathrm{c}$ & $34.94 \mathrm{~b}$ & $53.14 \mathrm{a}$ & $66.03 \mathrm{~b}$ \\
\hline Santa Rosa & $0.16 \mathrm{a}$ & $6.39 \mathrm{c}$ & $29.85 \mathrm{c}$ & $57.22 \mathrm{~b}$ & $75.79 \mathrm{a}$ & $95.73 \mathrm{~b}$ \\
\hline Own-rooted BRS Kampai & $0.23 \mathrm{a}$ & $10.59 \mathrm{~b}$ & $53.35 \mathrm{~b}$ & $109.10 \mathrm{a}$ & $132.21 \mathrm{a}$ & $159.15 \mathrm{a}$ \\
\hline $\mathrm{F}_{\text {rootstock }}$ & $1.5821^{\text {ns }}$ & $3.7420^{* *}$ & $5.6123^{* *}$ & $2.8613^{* *}$ & $1.7511^{\text {ns }}$ & $2.5240^{* *}$ \\
\hline $\bar{F}_{\text {block }}$ & $0.4805^{\text {ns }}$ & $1.6665^{\text {ns }}$ & $8.1787^{* *}$ & $5.2281^{* *}$ & $4.4636^{* *}$ & $7.6036^{* *}$ \\
\hline CV (\%) & 30.17 & 29.78 & 26.43 & 33.09 & 34.79 & 32.71 \\
\hline
\end{tabular}

Means followed by different letters in the column differ from each other by Scott-knott test. ${ }^{* *}$ significant at $99 \%$ confidence; ${ }^{\text {ns }}$ not significant. 
Fruit number per tree (FNT) was influenced by treatments in all evaluated crops, as well in the accumulated fruit number, where 'Capdeboscq' was found in the group with the lowest averages, together with 'Barrier', 'Ishtara' and 'Santa Rosa' (Table 4). Fruit weight (FW) was influenced by treatments in three crops (2017, 2018 and 2019), as well in the average of four crops (Table 5). So that, fruit yield per tree (FYT) was significantly influenced by the treatments in all the four crops evaluated (Table 6). 'Flordaguard' stood out for being one of those that presented the highest fruit number per tree, with good regularity along the years (Table 4) and fruit weight in the average or above than typical fruit weight of the cultivar (RASEIRA et al., 2010), which resulted in one of the largest annual and accumulated fruit yield per tree (Table 6). Another highlight was the Clone 15, for significantly increasing fruit weight of 'BRS Kampai' in all four evaluated crops (Table 5), maintaining a satisfactory amount of fruits per tree, which resulted in one of the highest average accumulated fruit yield (96.49 $\mathrm{kg}$ tree $^{-1}$ ) in the four crops (Table 6). Own-rooted trees also stood out positively, with good to excellent amount of fruit in all four crops (Table 4), fruit weight equivalent to those trees grafted on the traditional 'Capdeboscq' (Table 5), but with accumulated fruit yield per tree $31.5 \%$ higher (Table 6).

Table 4. Effects of clonal rootstocks used for 'BRS Kampai' peach and own-rooted trees (without rootstock) on fruit number per tree. Embrapa Clima Temperado, Pelotas-RS, Brazil.

\begin{tabular}{lccccc}
\hline \multirow{2}{*}{ Rootstock } & \multicolumn{5}{c}{ Fruit number per tree } \\
\cline { 2 - 6 } & 2016 & 2017 & 2018 & 2019 & $\begin{array}{c}\text { Accumulated } \\
(2016+2017+2018+2019)\end{array}$ \\
\hline Barrier & $107.75 \mathrm{a}$ & $119.25 \mathrm{~b}$ & $127.00 \mathrm{~b}$ & $187.75 \mathrm{~b}$ & $541.75 \mathrm{~b}$ \\
\hline Cadaman & $62.25 \mathrm{~b}$ & $109.75 \mathrm{~b}$ & $196.50 \mathrm{a}$ & $234.25 \mathrm{~b}$ & $602.75 \mathrm{a}$ \\
\hline GxN.9 & $60.25 \mathrm{~b}$ & $109.25 \mathrm{~b}$ & $161.50 \mathrm{~b}$ & $229.75 \mathrm{~b}$ & $560.75 \mathrm{a}$ \\
\hline Capdeboscq & $66.25 \mathrm{~b}$ & $114.00 \mathrm{~b}$ & $145.75 \mathrm{~b}$ & $169.50 \mathrm{~b}$ & $495.50 \mathrm{~b}$ \\
\hline Rigitano & $98.25 \mathrm{a}$ & $131.00 \mathrm{~b}$ & $224.00 \mathrm{a}$ & $134.25 \mathrm{~b}$ & $587.50 \mathrm{a}$ \\
\hline Clone 15 & $102.50 \mathrm{a}$ & $223.25 \mathrm{a}$ & $166.75 \mathrm{~b}$ & $174.25 \mathrm{~b}$ & $666.75 \mathrm{a}$ \\
\hline I-67-52-4 & $59.75 \mathrm{~b}$ & $155.50 \mathrm{a}$ & $209.25 \mathrm{a}$ & $243.50 \mathrm{~b}$ & $668.00 \mathrm{a}$ \\
\hline Tsukuba-1 & $110.00 \mathrm{a}$ & $112.25 \mathrm{~b}$ & $139.25 \mathrm{~b}$ & $235.00 \mathrm{~b}$ & $596.50 \mathrm{a}$ \\
\hline Tsukuba-2 & $88.75 \mathrm{a}$ & $131.25 \mathrm{~b}$ & $185.50 \mathrm{a}$ & $201.50 \mathrm{~b}$ & $607.00 \mathrm{a}$ \\
\hline Tsukuba-3 & $67.50 \mathrm{~b}$ & $125.25 \mathrm{~b}$ & $226.25 \mathrm{a}$ & $187.00 \mathrm{~b}$ & $606.00 \mathrm{a}$ \\
\hline Okinawa & $108.00 \mathrm{a}$ & $171.25 \mathrm{a}$ & $159.25 \mathrm{~b}$ & $202.75 \mathrm{~b}$ & $641.25 \mathrm{a}$ \\
\hline Flordaguard & $90.25 \mathrm{a}$ & $156.50 \mathrm{a}$ & $236.50 \mathrm{a}$ & $246.00 \mathrm{~b}$ & $729.25 \mathrm{a}$ \\
\hline Nemared & $55.75 \mathrm{~b}$ & $134.50 \mathrm{~b}$ & $209.00 \mathrm{a}$ & $364.00 \mathrm{a}$ & $763.25 \mathrm{a}$ \\
\hline Ishtara & $50.25 \mathrm{~b}$ & $72.25 \mathrm{~b}$ & $135.25 \mathrm{~b}$ & $108.50 \mathrm{~b}$ & $366.25 \mathrm{~b}$ \\
\hline Santa Rosa & $54.25 \mathrm{~b}$ & $106.00 \mathrm{~b}$ & $116.00 \mathrm{~b}$ & $189.50 \mathrm{~b}$ & $465.75 \mathrm{~b}$ \\
\hline Own-rooted 'BRS Kampai' & $92.00 \mathrm{a}$ & $142.75 \mathrm{~b}$ & $231.75 \mathrm{a}$ & $192.25 \mathrm{~b}$ & $658.75 \mathrm{a}$ \\
\hline F & $3.7684^{* *}$ & $2.6884^{* *}$ & $2.3046^{*}$ & $3.2930^{* *}$ & $3.0554^{* *}$ \\
\hline F & $5.7613^{* *}$ & $5.0371^{* *}$ & $7.2215^{* *}$ & $2.2900^{\mathrm{ns}}$ & $10.7913^{* *}$ \\
\hline CV (\%) & 28.38 & 31.37 & 29.77 & 30.34 & 18.91 \\
\hline
\end{tabular}

Means followed by different letters in the column differ from each other by Scott-knott test. * significant at $95 \%$ confidence; ** significant at $99 \%$ confidence; ${ }^{\text {ns }}$ not significant. 
Table 5. Effects of clonal rootstocks used for 'BRS Kampai' peach and own-rooted trees (without rootstock) on fruit weight (g). Embrapa Clima Temperado, Pelotas-RS, Brazil.

\begin{tabular}{lccccc}
\hline \multirow{2}{*}{ Rootstock } & \multicolumn{5}{c}{ Fruit weight (g) } \\
\cline { 2 - 6 } & 2016 & 2017 & 2018 & 2019 & $\begin{array}{c}\text { Average fruit } \\
\text { weight (four crops) }\end{array}$ \\
\hline Barrier & $104.45 \mathrm{a}$ & $110.10 \mathrm{~b}$ & $143.63 \mathrm{a}$ & $129.00 \mathrm{c}$ & $121.79 \mathrm{~b}$ \\
\hline Cadaman & $11.12 \mathrm{a}$ & $101.66 \mathrm{~b}$ & $101.40 \mathrm{c}$ & $94.33 \mathrm{~d}$ & $102.13 \mathrm{~d}$ \\
\hline GxN.9 & $119.40 \mathrm{a}$ & $102.29 \mathrm{~b}$ & $110.48 \mathrm{c}$ & $112.92 \mathrm{~d}$ & $111.27 \mathrm{c}$ \\
\hline Capdeboscq & $104.66 \mathrm{a}$ & $118.66 \mathrm{a}$ & $143.81 \mathrm{a}$ & $143.65 \mathrm{c}$ & $127.70 \mathrm{~b}$ \\
\hline Rigitano & $106.27 \mathrm{a}$ & $128.79 \mathrm{a}$ & $123.02 \mathrm{~b}$ & $158.13 \mathrm{~b}$ & $129.05 \mathrm{~b}$ \\
\hline Clone 15 & $119.26 \mathrm{a}$ & $122.66 \mathrm{a}$ & $138.18 \mathrm{a}$ & $196.96 \mathrm{a}$ & $144.27 \mathrm{a}$ \\
\hline I-67-52-4 & $103.63 \mathrm{a}$ & $99.07 \mathrm{~b}$ & $111.63 \mathrm{c}$ & $131.33 \mathrm{c}$ & $111.41 \mathrm{c}$ \\
\hline Tsukuba-1 & $110.80 \mathrm{a}$ & $112.55 \mathrm{a}$ & $117.97 \mathrm{~b}$ & $130.53 \mathrm{c}$ & $117.96 \mathrm{~b}$ \\
\hline Tsukuba-2 & $107.73 \mathrm{a}$ & $109.11 \mathrm{~b}$ & $122.79 \mathrm{~b}$ & $136.86 \mathrm{c}$ & $119.12 \mathrm{~b}$ \\
\hline Tsukuba-3 & $102.33 \mathrm{a}$ & $107.62 \mathrm{~b}$ & $121.02 \mathrm{~b}$ & $130.05 \mathrm{c}$ & $115.25 \mathrm{c}$ \\
\hline Okinawa & $98.47 \mathrm{a}$ & $107.00 \mathrm{~b}$ & $119.64 \mathrm{~b}$ & $134.37 \mathrm{c}$ & $114.87 \mathrm{c}$ \\
\hline Flordaguard & $106.15 \mathrm{a}$ & $114.32 \mathrm{a}$ & $126.75 \mathrm{~b}$ & $140.52 \mathrm{c}$ & $121.93 \mathrm{~b}$ \\
\hline Nemared & $78.96 \mathrm{a}$ & $98.48 \mathrm{~b}$ & $105.61 \mathrm{c}$ & $109.27 \mathrm{~d}$ & $98.08 \mathrm{~d}$ \\
\hline Ishtara & $112.52 \mathrm{a}$ & $96.44 \mathrm{~b}$ & $94.85 \mathrm{c}$ & $134.63 \mathrm{c}$ & $109.61 \mathrm{c}$ \\
\hline Santa Rosa & $115.74 \mathrm{a}$ & $86.29 \mathrm{~b}$ & $84.45 \mathrm{c}$ & $104.06 \mathrm{~d}$ & $97.64 \mathrm{~d}$ \\
\hline Own-rooted 'BRS Kampai' & $104.05 \mathrm{a}$ & $122.98 \mathrm{a}$ & $136.61 \mathrm{a}$ & $138.92 \mathrm{c}$ & $125.64 \mathrm{~b}$ \\
\hline F ${ }_{\text {rootstock }}$ & $1.6415^{\mathrm{ns}}$ & $3.9366^{* *}$ & $5.3901^{* *}$ & $11.1213^{* *}$ & $9.3478^{* *}$ \\
\hline F block & $0.9349^{\mathrm{ns}}$ & $1.3998^{\mathrm{ns}}$ & $3.9259^{*}$ & $1.0354^{\mathrm{ns}}$ & $1.1927^{\mathrm{ns}}$ \\
\hline CV (\%) & 13.92 & 10.43 & 12.40 & 10.61 & 6.80 \\
\hline
\end{tabular}

Means followed by different letters in the column differ from each other by Scott-knott test. * significant at $95 \%$ confidence; ** significant at $99 \%$ confidence; ${ }^{\text {ns }}$ not significant.

Table 6. Effects of clonal rootstocks used for 'BRS Kampai' peach and own-rooted trees (without rootstock) in fruit yield $\left(\mathrm{kg}\right.$ tree $\left.^{-1}\right)$. Embrapa Clima Temperado. Pelotas-RS.

\begin{tabular}{lccccc}
\hline \multirow{2}{*}{ Rootstock } & \multicolumn{5}{c}{ Fruit yield $\left(\mathrm{kg} \mathrm{tree}^{-1}\right)$} \\
\cline { 2 - 6 } & 2016 & 2017 & 2018 & 2019 & $\begin{array}{c}\text { Accumulated } \\
(2016+2017+2018+2019)\end{array}$ \\
\hline Barrier & $11.75 \mathrm{a}$ & $13.07 \mathrm{c}$ & $18.08 \mathrm{~b}$ & $25.34 \mathrm{~b}$ & $68.25 \mathrm{a}$ \\
\hline Cadaman & $6.91 \mathrm{~b}$ & $11.03 \mathrm{c}$ & $20.01 \mathrm{~b}$ & $21.92 \mathrm{~b}$ & $59.88 \mathrm{~b}$ \\
\hline GxN.9 & $6.78 \mathrm{~b}$ & $11.22 \mathrm{c}$ & $18.06 \mathrm{~b}$ & $26.30 \mathrm{~b}$ & $62.35 \mathrm{~b}$ \\
\hline Capdeboscq & $6.94 \mathrm{~b}$ & $12.81 \mathrm{c}$ & $20.68 \mathrm{~b}$ & $24.15 \mathrm{~b}$ & $64.57 \mathrm{~b}$ \\
\hline Rigitano & $10.45 \mathrm{a}$ & $16.74 \mathrm{~b}$ & $26.61 \mathrm{a}$ & $21.02 \mathrm{~b}$ & $74.81 \mathrm{a}$ \\
\hline Clone 15 & $12.29 \mathrm{a}$ & $27.33 \mathrm{a}$ & $22.68 \mathrm{a}$ & $34.19 \mathrm{a}$ & $96.49 \mathrm{a}$ \\
\hline I-67-52-4 & $6.22 \mathrm{~b}$ & $15.35 \mathrm{c}$ & $23.48 \mathrm{a}$ & $32.11 \mathrm{a}$ & $77.15 \mathrm{a}$ \\
\hline Tsukuba-1 & $12.46 \mathrm{a}$ & $12.40 \mathrm{c}$ & $16.42 \mathrm{~b}$ & $30.72 \mathrm{a}$ & $71.99 \mathrm{a}$ \\
\hline Tsukuba-2 & $9.61 \mathrm{a}$ & $14.35 \mathrm{c}$ & $23.08 \mathrm{a}$ & $27.22 \mathrm{~b}$ & $74.26 \mathrm{a}$ \\
\hline Tsukuba-3 & $6.91 \mathrm{~b}$ & $13.34 \mathrm{c}$ & $27.75 \mathrm{a}$ & $24.22 \mathrm{~b}$ & $72.23 \mathrm{a}$ \\
\hline Okinawa & $10.59 \mathrm{a}$ & $18.18 \mathrm{~b}$ & $19.19 \mathrm{~b}$ & $27.24 \mathrm{~b}$ & $75.19 \mathrm{a}$ \\
\hline Flordaguard & $9.64 \mathrm{a}$ & $17.61 \mathrm{~b}$ & $30.03 \mathrm{a}$ & $34.24 \mathrm{a}$ & $91.52 \mathrm{a}$ \\
\hline Nemared & $4.74 \mathrm{~b}$ & $13.11 \mathrm{c}$ & $22.07 \mathrm{a}$ & $39.20 \mathrm{a}$ & $79.11 \mathrm{a}$ \\
\hline Ishtara & $5.94 \mathrm{~b}$ & $6.83 \mathrm{c}$ & $12.84 \mathrm{~b}$ & $14.21 \mathrm{~b}$ & $39.81 \mathrm{c}$ \\
\hline Santa Rosa & $6.30 \mathrm{~b}$ & $9.11 \mathrm{c}$ & $9.40 \mathrm{~b}$ & $19.61 \mathrm{~b}$ & $44.41 \mathrm{c}$ \\
\hline Own-rooted 'BRS Kampai & $9.54 \mathrm{a}$ & $17.08 \mathrm{~b}$ & $32.01 \mathrm{a}$ & $26.30 \mathrm{~b}$ & $84.93 \mathrm{a}$ \\
\hline F ${ }_{\text {rootstock }}$ & $2.6795^{* *}$ & $4.7314^{* *}$ & $2.9059^{* *}$ & $2.5521^{* *}$ & $3.7326^{* *}$ \\
\hline F block & $4.5167^{* *}$ & $5.3048^{* *}$ & $6.9867^{* *}$ & $3.5032^{*}$ & $9.9958^{* *}$ \\
\hline CV (\%) & 35.60 & 29.75 & 32.76 & 29.39 & 21.74
\end{tabular}

Means followed by different letters in the column differ from each other by Scott-knott test. * significant at $95 \%$ confidence; ** significant at $99 \%$ confidence. 
When trees were still young (2016 and 2017), yield efficiency (YE) was significantly influenced by rootstocks. However, upon reaching the fourth year at the field (2018), this variable was no longer influenced by rootstocks (Table 7). Fruit yield per hectare (FYH) was also significantly affected by the rootstocks along the four evaluated crops (Table 8), with emphasis for Clone 15 and 'Flordaguard', which were the most productive in at least three crops. In accumulated fruit yield per hectare $(\mathrm{AFYH})$, statistical analysis allows to form three groups, with ten rootstocks ('Barrier', 'Rigitano', Clone 15, I-67-52-4, 'Tsukuba-1', 'Tsukuba-2', 'Tsukuba-3', 'Okinawa', 'Flordaguard' and 'Nemared') and own-rooted trees in group with the highest averages, three rootstocks in intermediate group ('Cadaman', G x N.9 and 'Capdeboscq') and two rootstocks ('Ishtara' and 'Santa Rosa') in the group with the lowest accumulated fruit yields per hectare.

Table 7. Effects of clonal rootstocks used for 'BRS Kampai' peach and own-rooted trees (without rootstock) on yield efficiency $\left(\mathrm{kg} \mathrm{cm}^{2}\right)$. Embrapa Clima Temperado, Pelotas-RS, Brazil.

\begin{tabular}{lcccc}
\hline \multirow{2}{*}{ Rootstock } & \multicolumn{4}{c}{ Yield efficiency $\left(\mathrm{kg} \mathrm{cm}^{2}\right)$} \\
\cline { 2 - 5 } Barrier & 2016 & 2017 & 2018 & 2019 \\
\hline Cadaman & $0.3616 \mathrm{a}$ & $0.2716 \mathrm{a}$ & $0.1988 \mathrm{a}$ & $0.2790 \mathrm{a}$ \\
\hline GxN.9 & $0.1723 \mathrm{~b}$ & $0.1628 \mathrm{~b}$ & $0.2907 \mathrm{a}$ & $0.2404 \mathrm{a}$ \\
\hline Capdeboscq & $0.1753 \mathrm{~b}$ & $0.1896 \mathrm{~b}$ & $0.2046 \mathrm{a}$ & $0.2868 \mathrm{a}$ \\
\hline Rigitano & $0.1613 \mathrm{~b}$ & $0.1574 \mathrm{~b}$ & $0.2110 \mathrm{a}$ & $0.2233 \mathrm{a}$ \\
\hline Clone 15 & $0.2308 \mathrm{~b}$ & $0.3211 \mathrm{a}$ & $0.4125 \mathrm{a}$ & $0.1912 \mathrm{a}$ \\
\hline I-67-52-4 & $0.1671 \mathrm{~b}$ & $0.3146 \mathrm{a}$ & $0.2011 \mathrm{a}$ & $0.2600 \mathrm{a}$ \\
\hline Tsukuba-1 & $0.1949 \mathrm{~b}$ & $0.2925 \mathrm{a}$ & $0.3210 \mathrm{a}$ & $0.3559 \mathrm{a}$ \\
\hline Tsukuba-2 & $0.3115 \mathrm{a}$ & $0.1990 \mathrm{~b}$ & $0.2002 \mathrm{a}$ & $0.2388 \mathrm{a}$ \\
\hline Tsukuba-3 & $0.2993 \mathrm{a}$ & $0.2962 \mathrm{a}$ & $0.3410 \mathrm{a}$ & $0.3325 \mathrm{a}$ \\
\hline Okinawa & $0.2222 \mathrm{~b}$ & $0.2145 \mathrm{~b}$ & $0.2725 \mathrm{a}$ & $0.2517 \mathrm{a}$ \\
\hline Flordaguard & $0.2505 \mathrm{a}$ & $0.2891 \mathrm{a}$ & $0.2362 \mathrm{a}$ & $0.2515 \mathrm{a}$ \\
\hline Nemared & $0.1587 \mathrm{~b}$ & $0.1838 \mathrm{~b}$ & $0.3150 \mathrm{a}$ & $0.1954 \mathrm{a}$ \\
\hline Ishtara & $0.1375 \mathrm{~b}$ & $0.1711 \mathrm{~b}$ & $0.2475 \mathrm{a}$ & $0.3425 \mathrm{a}$ \\
\hline Santa Rosa & $0.2725 \mathrm{a}$ & $0.1979 \mathrm{~b}$ & $0.2500 \mathrm{a}$ & $0.2420 \mathrm{a}$ \\
\hline Own-rooted 'BRS Kampai' & $0.2056 \mathrm{~b}$ & $0.1672 \mathrm{~b}$ & $0.1231 \mathrm{a}$ & $0.2147 \mathrm{a}$ \\
\hline F & $0.1806 \mathrm{~b}$ & $0.1812 \mathrm{~b}$ & $0.2588 \mathrm{a}$ & $0.1707 \mathrm{a}$ \\
\hline Footstock & $2.2736^{*}$ & $1.9565^{*}$ & $1.2255^{\text {ns }}$ & $1.4325^{\text {ns }}$ \\
\hline CV (\%) & $0.8967^{\text {ns }}$ & $2.1022^{\text {ns }}$ & $1.0861^{\text {ns }}$ & $2.8189^{*}$ \\
\hline
\end{tabular}

Means followed by different letters in the eolumn differ from eaeh other by Seott-knott test. * signifieant at $95 \%$ eonfidenee; ${ }^{\text {Hs }}$ not signifieant. 
Table 8. Effects of clonal rootstocks used for 'BRS Kampai' peach and own-rooted trees (without rootstock) on fruit yield per hectare $\left(\mathrm{t} \mathrm{ha}^{-1}\right)$. Embrapa Clima Temperado, Pelotas-RS, Brazil.

\begin{tabular}{lccccc}
\hline \multirow{2}{*}{ Rootstock } & \multicolumn{5}{c}{ Fruit yield per hectare $\left(\mathrm{t} \mathrm{ha} \mathrm{H}^{-1}\right)$} \\
\cline { 2 - 6 } & 2016 & 2017 & 2018 & 2019 & $\begin{array}{c}\text { Accumulated } \\
\text { Barrier }\end{array}$ \\
\hline Cadaman & $6.52 \mathrm{a}$ & $7.26 \mathrm{c}$ & $10.04 \mathrm{~b}$ & $14.06 \mathrm{~b}$ & $37.88 \mathrm{a}$ \\
\hline GxN.9 & $3.76 \mathrm{~b}$ & $6.13 \mathrm{c}$ & $11.11 \mathrm{~b}$ & $12.16 \mathrm{~b}$ & $33.23 \mathrm{~b}$ \\
\hline Capdeboscq & $6.23 \mathrm{c}$ & $10.03 \mathrm{~b}$ & $14.60 \mathrm{~b}$ & $34.61 \mathrm{~b}$ \\
\hline Rigitano & $3.85 \mathrm{~b}$ & $7.11 \mathrm{c}$ & $11.48 \mathrm{~b}$ & $13.40 \mathrm{~b}$ & $35.84 \mathrm{~b}$ \\
\hline Clone 15 & $5.80 \mathrm{a}$ & $9.30 \mathrm{~b}$ & $14.76 \mathrm{a}$ & $11.67 \mathrm{~b}$ & $41.53 \mathrm{a}$ \\
\hline I-67-52-4 & $6.82 \mathrm{a}$ & $15.17 \mathrm{a}$ & $12.59 \mathrm{a}$ & $18.98 \mathrm{a}$ & $53.55 \mathrm{a}$ \\
\hline Tsukuba-1 & $3.45 \mathrm{~b}$ & $8.52 \mathrm{c}$ & $13.03 \mathrm{a}$ & $17.82 \mathrm{a}$ & $42.82 \mathrm{a}$ \\
\hline Tsukuba-2 & $6.92 \mathrm{a}$ & $6.88 \mathrm{c}$ & $9.11 \mathrm{~b}$ & $17.05 \mathrm{a}$ & $39.96 \mathrm{a}$ \\
\hline Tsukuba-3 & $5.33 \mathrm{a}$ & $7.96 \mathrm{c}$ & $12.81 \mathrm{a}$ & $15.11 \mathrm{~b}$ & $41.21 \mathrm{a}$ \\
\hline Okinawa & $3.84 \mathrm{~b}$ & $7.40 \mathrm{c}$ & $15.40 \mathrm{a}$ & $13.44 \mathrm{~b}$ & $40.09 \mathrm{a}$ \\
\hline Flordaguard & $5.87 \mathrm{a}$ & $10.09 \mathrm{~b}$ & $10.65 \mathrm{~b}$ & $15.12 \mathrm{~b}$ & $41.73 \mathrm{a}$ \\
\hline Nemared & $5.35 \mathrm{a}$ & $9.77 \mathrm{~b}$ & $16.67 \mathrm{a}$ & $19.01 \mathrm{a}$ & $50.80 \mathrm{a}$ \\
\hline Ishtara & $2.63 \mathrm{~b}$ & $7.28 \mathrm{c}$ & $12.25 \mathrm{a}$ & $21.76 \mathrm{a}$ & $43.91 \mathrm{a}$ \\
\hline Santa Rosa & $3.30 \mathrm{~b}$ & $3.79 \mathrm{c}$ & $7.12 \mathrm{~b}$ & $7.89 \mathrm{~b}$ & $22.10 \mathrm{c}$ \\
\hline Own-rooted 'BRS Kampai' & $3.50 \mathrm{~b}$ & $5.06 \mathrm{c}$ & $5.21 \mathrm{~b}$ & $10.88 \mathrm{~b}$ & $24.65 \mathrm{c}$ \\
\hline F & $5.30 \mathrm{a}$ & $9.48 \mathrm{~b}$ & $17.77 \mathrm{a}$ & $14.60 \mathrm{~b}$ & $47.14 \mathrm{a}$ \\
\hline Footstock & $2.6774^{* *}$ & $4.7305^{* *}$ & $2.9071^{* *}$ & $2.5524^{* *}$ & $3.7327^{* *}$ \\
\hline CV (\%) & $4.5165^{* *}$ & $5.3027^{* *}$ & $6.9855^{* *}$ & $3.5041^{*}$ & $9.9972^{* *}$ \\
\hline
\end{tabular}

Means followed by different letters in the column differ from each other by Scott-knott test. * significant at $95 \%$ confidence; ** significant at $99 \%$ confidence.

With evaluations carried out from 2014 to 2019, rootstocks influenced all variables, in practically every year (Tables 2, 3, 4, 5, 6, 7 and 8). These significant effects are attributed to the several rootstocks (15) and considerable genetic variability tested, composed of $P$. persica (eight genotypes), P. mume (two genotypes), $P$. salicina (one genotype), four rootstock interspecific hybrids, in addition to own-rooted trees, which allowed to obtain a wider range of effects. Like what has been done for many decades in other fruit species (such as citrus, apple, pear, passion fruit and grape, among others), strategy to expand genetic variability to be tested as a peach rootstock in Southern Brazil is also necessary. The traditional 'Capdeboscq' and 'Aldrighi' (P. persica), widely used as rootstocks until the 1980 s, do not provide resistance to root-knot nematodes, soil waterlogging or PTSL syndrome (MAUCH et al., 1991; GUERRA et al., 1992; CARNEIRO et al., 1998; SOMAVILLA, 2008; MAYER et al., 2014a). Several interspecific hybrids and species are commercially used as peach rootstocks in Europe, mainly to tolerate soil water stress, physical or chemical problems. However, in the United States, $P$. persica cultivars (Lovell, Halford, Nemaguard, Nemared, Bailey and Guardian ${ }^{\circledR}$ ) still predominate (REIGHARD; LORETI, 2008; DUVAL, 2015).

The vigor of the rootstocks is a relative characteristic, because it depends, in addition to the genetic effects, on soil and climatic conditions, cultural treatments, fertilization (mainly nitrogen), spacing between trees, number of tested rootstocks and the genetic variability being evaluated in the trial, the reference rootstock and the scion/rootstock interaction (LAYNE, 1987; LORETI and MASSAI, 2002; MAYER et al., 2006; REIGHARD; LORETI, 2008; DAVIES Jr., 2017). Rootstock vigor can be assessed by trunk diameter, TCSA, tree height, canopy volume, fresh and dry pruning mass (DE ROSSI et al., 2004; REIGHARD and LORETI, 2008; BARRETO et al., 2017). In the present study, although Clone 15 (P. mume) had one of the largest trunk diameter and TCSA, trees on this rootstock had shoots with a shorter internodes and compact tree shape, aspects also conferred by trees on 'Rigitano' (P. mume). Both rootstocks were also the only ones to produce root suckers all over the years (until three per tree/year) and trees were apparently more susceptible to leaf diseases and phytotoxicity. As both induces a more compact tree, Clone 15 and 'Rigitano' are interesting for high density ( $>800$ trees per hectare), especially in regions with low chilling accumulation $\left(<50\right.$ chilling hours $\left.\leq 7.2^{\circ} \mathrm{C}\right)$ where the sun usually causes burns on scaffolds (MAYER et al., 2014b). At those edaphoclimatic conditions (Jaboticabal region, São Paulo State), 'Aurora-1' peach on Clone 15 or on 'Rigitano' with micro-sprinkler irrigation system showed satisfactory root anchorage and increased fruit 
size and weight, when compared to those on 'Okinawa' (MAYER et al., 2006; MAYER et al., 2007; MATHIAS et al., 2008). So, rootstock recommendation must be specific for each scion/rootstock combination and edaphoclimatic condition.

The 'Flordaguard' was the only rootstock having the highest fruit number per tree in three harvests (2016, 2017 and 2018), which is attributed to the high tree size it induces to the scion. The G x N.9, 'Ishtara' and 'Santa Rosa', as well as the traditional 'Capdeboscq', were negative highlights, with the lowest amounts of fruit per tree in all the four evaluated crops (Table 4). By another criterion for defining hand fruit thinning (leaving 5 fruit/ $\mathrm{cm}^{2}$ of TCSA) (PEREIRA; RASEIRA, 2014b), the data also showed that all scion/rootstock combinations had potential to support a greater amount of fruit per tree. Thus, by this criterion and dividing the values showed in Table 4 by values in Table 3 , this index varied from 1.21 fruits $/ \mathrm{cm}^{2}$ (on own-rooted trees in 2019) up to 3.21 fruits/ $\mathrm{cm}^{2}$ (on 'Barrier' in 2016). The annual averages, with all rootstocks combined (data not shown), were close to 2 fruits $/ \mathrm{cm}^{2}$ of TCSA, indicating that all scion/rootstock combinations could withstand double fruit number per tree, in the four evaluated crops, without impairing fruit quality. Smaller amouts of fruit in the lower tree part may explain the lower yield per tree in relation to their potential (based on TCSA), although this was observed in all treatments.

The fruit weight (FW) is a function of fruit load, tree vigor and edaphoclimatic conditions (mainly soil temperature and soil humidity) before harvest (LAYNE, 1994). Fruit weight (Table 5) was not influenced by rootstocks when trees were still young, with two years old in 2016. However, with increasing age, significant differences occurred, with the highest values seen on six treatments in 2017, four in 2018 and only one treatment in 2019. Clone 15 being the only one to confer the highest fruit weight in all four crops, proving an important characteristic that species (P.mume) can confer to the scion when used as a rootstock for peach, since selected clones and vegetative propagation be used (MAYER et al., 2006; MATHIAS et al., 2008; MAYER et al., 2015). The common fruit weight of 'BRS Kampai', when budded on 'Capdeboscq' and cultivated in Pelotas-RS, is between 112 and $127 \mathrm{~g}$ (RASEIRA et al., 2010). In the present study, fruit weight was increased by up to $37 \%$ in trees budded on Clone 15, compared to fruits produced on 'Capdeboscq' (in 2019), with the same amount of fruits per tree (Table 4). The greater fruit weight conferred by Clone 15 and 'Rigitano' compared to those produced on 'Okinawa', has already been reported for 'BRS Libra', in Chapecó, Santa Catarina State, with fruit weight increments of $25 \%$ to 38 $\%$ (SANTANA et al., 2020) and also for 'Aurora-1', in Vista Alegre do Alto, São Paulo State, with increases of $18 \%$ to $30 \%$ in fruit weight, beyond anticipation of five to seven days for fruit ripeness (MATHIAS et al., 2008). The greater efficiency on uptake and translocation of nutrient and water by the rootstock to the scion is usually the reason for fruit weight increasing (REIGHARD and LORETI, 2008), aspects that also are being investigated in these P.mume clones.

Fruit yield per tree (FYT) is a function of fruit set, thinning intensity, fruit size, fruit number per tree, tree size and environmental conditions (mainly air temperature and rainfall) that precede harvest (LAYNE, 1994). When observing yields per tree and per hectare (Tables 6 and 8), it turns out that 'Rigitano', Clone 15, I-67-52-4, 'Tsukuba-1', 'Tsukuba-2', 'Flordaguard' and 'Nemared' rootstocks, as well as own-rooted trees, presented significantly higher FYT averages than the traditional 'Capdeboscq', in most evaluated years. At four-years old (2018 season), yield per hectare conferred by several of the rootstocks tested was higher than the yield average obtained in commercial orchards in Pelotas$\mathrm{RS}$, which is $11.1 \mathrm{t} \mathrm{ha}^{-1}$ (IBGE, 2020). Yields per hectare greater than produced on 'Capdeboscq' were obtained from seven rootstocks in 2018 ('Rigitano', Clone 15, I-67-52-4, 'Tsukuba-2', 'Tsukuba-3', 'Flordaguard' or 'Nemared'), beyond own-rooted trees. With five-years old (2019 season), yields per hectare increased and, on Clone 15, I-67-52-4, 'Tsukuba-1', 'Flordaguard' and 'Nemared' rootstocks, were significantly higher in relation to 'Capdeboscq'. These data prove that cultivars or selections developed for rootstock purpose can increase yields per tree and per hectare, as compared in relation to the regional reference 'Capdeboscq' used as a rootstock for many years. Rootstocks with desirable characteristics such as better adaptation and induction of greater yield, can offer an important contribution to regional peach production, as this objective can be achieved without increasing nursery trees prices, due to rootstock change.

The 'Barrier' rootstock, although had one of the highest accumulated yields per tree and per hectare in four years (Tables 6 and 8), it producing fruits with low weight (Table 5) and early bud breaking of the scion at end Summer, undesirable aspects that were also observed in trees on 'Cadaman'. Trees budded on G x N.9 showed typical symptoms of "translocated" graft incompatibility at the second year, bud drop and early defoliation, with soft dormant shoots in Winter and few standard fruit at harvest, as well as the symptoms described by Davies Jr. (2017). 'Ishtara' and 'Santa Rosa' also induce to the scion little yield and low fruit quality, in addition to symptoms of "translocated" graft incompatibility, but these symptoms were not as pronounced, as observed in G x N.9, and were visible only after three-years old. Translocated graft incompatibility of 'Myrobalan 29C' (P.cerasifera) and 'Marianna 2624' (P.cerasifera x P.munsoniana) with 'BRS Libra', 'BRS Kampai', 'Maciel' and 'Jade' scion peaches had already been detected before one-year old 
trees in the field (NEVES et al., 2017; GONÇALVES et al., 2019; SANTANA et al., 2020).

An alternative that eliminates the need of grafting is the use of own-rooted nursery trees. This type of tree has interesting advantages in the nursery phase, such as the shorter time to produce nursery trees and it eliminate all operations inherent to the grafting process (COUVILLON, 1985; DAVIES Jr., 2017; MAYER et al., 2018). In the field, own-rooted trees can delay bloom, increase fruit size, fruit weight, yield per tree and per hectare of some peach and nectarine cultivars in soil without physical, chemical or biological restrictions (COUVILLON, 1985; GONÇALVES et al., 2019; JIMENEZ et al., 2020; SANTANA et al., 2020). The 'BRS Kampai' own-rooted trees showed high vigor, with high TCSA (Table 3 ), fruit number per tree and fruit weight equivalent to the best rootstocks (Tables 4 and 5). However, this kind of tree have a more vertical growth habit and greater production of sucker shoots, which makes more difficult to manage trees on the "open-vase" trainning system during summer and winter pruning. The accumulated yield per tree of own-rooted trees in all four seasons was one of the greatest $\left(84.93 \mathrm{~kg}\right.$ tree $\left.^{-1}\right)$, similar to those obtained in the best rootstocks (Table 6), which resulted in $47.14 \mathrm{t} \mathrm{ha}^{-1}$ average of accumulated yield (Table 8). Thus, in the four harvests, the accumulated yield per hectare in own-rooted trees was $31.5 \%$ higher, compared to the trees budded on 'Capdeboscq'. Own-rooted peach trees can be a desirable alternative, as long as they are grown in soils that do not present problems with nematodes and without physicalchemical limitations (COUVILLON, 1985). Root quality of own-rooted trees is essential for initial setting and survival at the field, since trees with poor root system can result in $30 \%$ mortality in the first year. In addition, it become more susceptible to soil moisture at Fall, which is unfavorable for acclimatization to cold, as described by Layne (1994).

In the present study, the preservation of intact root system of the nursery trees at planting contributed for an excellent initial setting and growth at the field, with $100 \%$ tree survival of all 16 treatments. Trees had excellent anchorage and there were no falling problems. Nursery trees with satisfactory morphological pattern, provided by efficient rootstock and scion propagation methods and by a good nursery tree production system (bagged or potted trees), is an essential condition for successful orchard establishment and satisfactory yields (MAYER et al., 2019). Approximately $70 \%$ of total non-structural carbohydrate content in dormant young peach trees is found in roots and, of this total, more than $80 \%$ of which in thin roots (WEIBEL et al., 2011), which proves the importance of a good root system for initial establishment at the field. According to Layne (1994), the main cause of tree mortality in the first year is the low quality of nursery trees, especially on those with poor root development.
Vegetative propagation of rootstocks is an efficient and quick way to perpetuate all selected characteristics in the cultivar's development (DAVIES Jr. et al., 2017), besides not presenting disadvantages of seed propagation (MILLER et al., 1989; BECKMAN, 1998). For these reasons, vegetative propagation of Prunus rootstocks by the softwood cuttings, as adopted for production of nursery trees in this research, represents a good prospect for use in commercial orchards.

\section{Conclusions}

In the adopted experimental conditions, we conclude that 'Barrier' (P. persica $\times$ P. davidiana), 'Cadaman' (P. persica x P. davidiana), G x N.9 (P. persica $\mathrm{x} P$. dulcis $)$, 'Ishtara' $[(P$. cerasifera $\mathrm{x} P$. salicina $) \times(P$. cerasifera $\mathrm{x} P$. persica)] and 'Santa Rosa' $(P$. salicina $)$ do not have potential as a rootstock of 'BRS Kampai' peach due irregular bud breaking of scion, small fruits and/or low fruit yield. Clone 15 (P. mume) stands out for increasing fruit weight and maintaining satisfactory fruit yield per tree. 'Flordaguard' $(P$. persica) is the best rootstock tested, which is vigorous, do not present any undesirable agronomic characteristics and induce the highest yields per tree and per hectare, exceeding $50 \mathrm{tha}^{-1}$ in accumulated yield. Own-rooted trees of 'BRS-Kampai' peach have technical feasibility, as long as pruning is properly carried out.

\section{Acknowledgment}

Thanks to Empresa Brasileira de Pesquisa Agropecuária (Embrapa) [Project 02.13.06.001.00.00], Coordenação de Aperfeiçoamento de Pessoal de Nível Superior (CAPES) for scholarship grant to F.M. Roth, and Conselho Nacional de Desenvolvimento Científico e Tecnológico (CNPq) for research grant DT-2 to N.A. Mayer and research grant PQ to L.E.C. Antunes.

\section{References}

AGRIANUAL. Pêssego. São Paulo: FNP Consultoria \& Comércio, 2020. p.347-353.

AGROMET - Laboratório de Agrometeorologia. Dados climáticos. Brasília, DF: EMBRAPA, 2020. Disponível em: http://agromet.cpact.embrapa.br. Acesso em: 26 jun. 2020.

BARRETO, C.F.; KIRINUS, M.B.M.; SILVA, P.S.; SCHIAVON, C.R.; ROMBALDI, C.V.; MALGARIM, M.B.; FACHINELLO, J.C. Agronomic performance of the Maciel peach with different rootstocks. Semina: Ciências Agrárias, Londrina, v.38, n.3, p.1217-1228, 2017. 
BECKMAN, T.G. Outcrossing in a diverse peach rootstock seed block. Fruit Varieties Journal, University Park, v.52, n.2, p.100-103, 1998.

BECKMAN, T.G.; LANG, G.A. Rootstock breeding for stone fruits. Acta Horticulturae, Leuven, n.622, p.531550,2003

BUSSI, C.; BESSET, J.; GIRARD, T. Effects of peach or hybrid rootstocks on growth and cropping of two cultivars of peach trees (Emeraude and Zephyr). Fruits, Paris, v.57, n.4, p.249-255, 2002.

CABRERA, D.; RODRIGUEZ, P. Portainjertos para duraznero. In: BARÁIBAR, J.S. Manual del duraznero: la planta y la cosecha. Montevideo: INIA, 2014. p.61-83. (Boletín de Divulgación, 108).

CAMPO DALL'ORTO, F.A.; OJIMA, M.; BARBOSA, W.; MARTINS, F.P. O nanismo do pessegueiro induzido pela enxertia no damasqueiro-japonês. Pesquisa Agropecuária Brasileira, Brasília, DF, v.27, p.517-521, 1992.

CANTERI, M.G.; ALTHAUS, R.A.; VIRGENS FILHO, J.S.; GIGLIOTI, E.A.; GODOY, C.V. SASM - Agri: Sistema para análise e separação de médias em experimentos agrícolas pelos métodos Scott - Knott, Tukey e Duncan. Revista Brasileira de Agrocomputação, Ponta Grossa, v.1, n.2, p.18-24, 2001.

CARNEIRO, R.M.D.G.; CAMPOS, A.D.; PEREIRA, J.F.M.; RASEIRA, M.C.B. Avaliação de porta-enxertos de Prunus quanto à suscetibilidade ao nematóide anelado e ao conteúdo de enzimas fenol oxidases. Nematologia Brasileira, Campinas, v.22, n.1, p.32-38, 1998.

COUVILLON, G.A. Propagation and performance of inexpensive peach trees from cuttings for high density peach plantings. Acta Horticulturae, Leuven, n.173, p.271-281, 1985.

CQFS - Comissão de Química e Fertilidade do Solo. Manual de adubação e de calagem para os Estados do Rio Grande do Sul e de Santa Catarina. 10.ed. Porto Alegre: Núcleo Regional Sul - Sociedade Brasileira de Ciência do Solo, 2004. 400 p.

CQFS - Comissão de Química e Fertilidade do Solo. 2016. Manual de calagem e adubação para os Estados de Rio Grande do Sul e de Santa Catarina. 11.ed. Frederico Westphalen: Núcleo Regional Sul - Sociedade Brasileira de Ciência do Solo, 2016. 376 p.
DAVIES JR., F.T.; GENEVE, R.L.; WILSON, S.B. Plant propagation: principles and practices. $9^{\text {th }} \mathrm{ed}$. New Jersey: Pearson, 2017. 1024p.

DE ROSSI, A.; FACHINELLO, J.C.; RUFATO, L.; PARISOTTO, E.; PICOLOTTO, L.; KRUGER, L.R. Comportamento do pessegueiro 'Granada' sobre diferentes porta-enxertos. Revista Brasileira de Fruticultura, Jaboticabal, v.26, n.3, p.446-449, 2004.

DI VITO, M.; BATTISTINI, A.; CATALANO, L. Response of Prunus rootstocks to root-knot (Meloidogyne spp.) and root-lesion (Pratylenchus vulnus) nematodes. Acta Horticulturae, Leuven, n.592, p.663-668, 2002.

DUVAL, H. Use of Prunus genetic diversity for peach rootstocks. Acta Horticulturae, Leuven, n.1084, p.277282, 2015.

FERGUSON, J.; CHAPARRO, J. Rootstocks for Florida peaches, nectarines, and plums. Gainesville: University of Florida, IFAS Extension, 2008. Disponível em: https:// journals.flvc.org/edis/article/view/117047. Acesso em: 24 ago. 2020.

FINARDI, N.L. Morte de plantas de pessegueiro e ameixeira por asfixia do sistema radicular. HortiSul, Bagé, v.3, n.3, p.18-26, 1995.

FINARDI, N.L. Método de propagação e descrição de porta-enxertos. In: MEDEIROS, C.A.B.; RASEIRA, M.C.B. A cultura do pessegueiro. Brasília, DF: EmbrapaSPI; Pelotas: Embrapa-CPACT, 1998. p.100-129.

GALARÇA, S.P.; FACHINELLO, J.C.; BETEMPS, D.L.; HOFFMANN, A.; MARODIN, G.A.B.; PRETTO, F.; NUNES, F.S.; DIAS, F.P. Crescimento e desenvolvimento de pessegueiros 'Chimarrita' e 'Maciel' sobre diferentes porta-enxertos e locais de cultivo. Ciência Rural, Santa Maria, v.43, n.2, p.219-224, 2013.

GONÇALVES, E.D.; MONTEIRO, V.F.C.; MAYER, N.A.; MOURA, P.H.A.; ALVARENGA, A.A.; ANTUNES, L.E.C.; TREVISAN, R.; PÁDUA, J.G. Desempenho de pessegueiro 'BRS Libra' autoenraizado e enxertado sobre porta-enxertos clonais em Minas Gerais. Revista Brasileira de Ciências Agrárias, Recife, v.14, n.1, e5610, 2019.

GUERRA, L.J.; FINARDI, N.L.; SANTOS FILHO, B.G.; PETERS, J.A. Influência do alagamento na mortalidade do pessegueiro e da ameixeira. Pesquisa Agropecuária Brasileira, Brasília, DF, v.27, n.3, p.499-508, 1992. 
IBGE - Instituto Brasileiro de Geografia e Estatística. Censo Agro 2017. Pêssego. Disponível em: https://censos.ibge. gov.br/agro/2017/templates/censo agro/resultadosagro/ agricultura. $h$ tml ?localidade $=43 \&$ tema $=78300$. Acesso em: 19 abr. 2020

JIMENES, I.M.; MAYER, N.A.; DIAS, C.T.S.; SILVA, S.R. Initial performance of own-rooted and buddes 'Sunraycer' nectarine plants. Pesquisa Agropecuária Brasileira, Brasília, DF, v.55, e00777, 2020.

LAYNE, R.E.C. Peach rootstocks. In: ROM, R.C.; CARLSON, R.F. Rootstocks for fruit crops. Hoboken: John Wiley \& Sons, 1987. p.185-216.

LAYNE, R.E.C. Prunus rootstock affect long-term orchard performance of 'Redhaven' peach on brookston clay loam. HortScience, Alexandria, v.29, n.3, p.167-171, 1994.

LORETI, F.; MASSAI, R. The high density peach planting system: present status and perspectives. Acta Horticulturae, Leuven, n.592, p.377-390, 2002.

MATHIAS, C.; MAYER, N.A.; MATTIUZ, B.; PEREIRA, F.M. Efeito de porta-enxertos e espaçamentos entre plantas na qualidade de pêssegos 'Aurora-1'. Revista Brasileira de Fruticultura, Jaboticabal, v.30, n.1, p.165-170, 2008.

MAUCH, C.H.; MAUCH, N.; FINARDI, N.L. Reações de pessegueiros e da ameixeira ao nematóide das galhas Meloidogyne incognita. Nematologia Brasileira, Piracicaba, v.15, n.1, p.59-67, 1991.

MAYER, N.A.; PEREIRA, F.M.; SANTOS, J.M. Reação de clones de umezeiro (Prunus mume Sieb. et Zucc.) e cultivares de pessegueiro a Meloidogyne javanica (Treub, 1885) Chitwood, 1949. Revista Brasileira de Fruticultura, Jaboticabal, v.25, n.1, p.181-183, 2003.

MAYER, N.A.; PEREIRA, F.M.; SANTOS, J.M. Resistência de clones de umezeiro e cultivares de pessegueiro a Meloidogyne incognita (Nemata: Heteroderidae). Revista Brasileira de Fruticultura, Jaboticabal, v.27, n.2, p.335-337, 2005.

MAYER, N.A.; PEREIRA, F.M.; KOBA, V.Y. Desenvolvimento inicial no campo de pessegueiros 'Aurora-1' enxertados em clones de umezeiro e 'Okinawa' propagados por estacas herbáceas. Revista Brasileira de Fruticultura, Jaboticabal, v.28, n.2, p.231-235, 2006.
MAYER, N.A.; PEREIRA, F.M.; BARBOSA, J.C.; KOBA, V.Y. Distribuição do sistema radicular de porta-enxertos de umezeiro enxertados com o pessegueiro 'Aurora-1'. Pesquisa Agropecuária Brasileira, Brasília, DF, v.42, n.7, p.965-973, 2007.

MAYER, N.A.; UENO, B. A morte-precoce do pessegueiro e suas relações com porta-enxertos. Pelotas: Embrapa Clima Temperado, 2012. 42p. (Documentos, 359).

MAYER, N.A.; UENO, B.; FISCHER, C.; MIGLIORINI, L.C. Propagação vegetativa de frutíferas de caroço por estacas herbáceas em escala comercial. Pelotas: Embrapa Clima Temperado, 2013. 55p. (Boletim de Pesquisa e Desenvolvimento, 195).

MAYER, N.A.; BIANCHI, V.J.; CASTRO, L.A.S. Portaenxertos. In: RASEIRA, M.C.B.; PEREIRA, J.F.M.; CARVALHO, F.L.C. Pessegueiro. Brasília: Embrapa, 2014a. p.173-223.

MAYER, N.A.; PEREIRA, F.M.; REIGHARD, G.L. Prunus mume clones as rootstocks for 'Aurora-1' peach in São Paulo State, Brazil and planting density. Acta Horticulturae, Leuven, n.1058, p.619-626, 2014b.

MAYER, N.A.; UENO, B.; REIGHARD, G.L.

Selection of Prunus mume as rootstocks for peaches on PTSL site. Acta Horticulturae, Leuven, n.1084, p.8996, 2015.

MAYER, N.A.; BIANCHI, V.J.; FELDBERG, N.P.; MORINI, S. Advances in peach, nectarine and plum propagation. Revista Brasileira de Fruticultura, Jaboticabal, v.39, n.4, e-355, 2017.

MAYER, N.A.; UENO, B.; RICKES, T.B. Produção rápida de mudas de pessegueiro. Pelotas: Embrapa Clima Temperado, 2018. 20p. (Documentos, 469).

MAYER, N.A.; UENO, B.; NEVES, T.R.; RICKES, T.B. Cinco anos de avaliações dos efeitos de porta-enxertos sobre a produção, produtividade e eficiência produtiva do pessegueiro 'Maciel'. Revista de La Facultad de Agronomia, La Plata, v.118, n.2, p.1-11, 2019.

MILLER, P.J.; PARFITT, D.E.; WEINBAUM, S.A. Outcrossing in peach. HortScience, Alexandria, v.24, n.2, p.359-360, 1989. 
NACHTIGAL, J.C. Obtenção de porta-enxertos 'Okinawa' e de mudas de pessegueiro (Prunus persica (L.) Batsch) utilizando métodos de propagação vegetativa. 1999. Tese (Doutorado em Agronomia) - Faculdade de Ciências Agrárias e Veterinárias, Universidade Estadual Paulista, Jaboticabal, 1999.

NEVES, T.R.; MAYER, N.A.; UENO, B. Graft incompatibility in Prunus spp. preceded by SPAD index reduction. Semina: Ciências Agrárias, Londrina, v.38, n.2, p.635-648, 2017.

PEREIRA, F.M.; MAYER, N.A.; CAMPO DALL'ORTO, F.A. 'Rigitano': nova cultivar de umezeiro para porta-enxerto de pessegueiro. Revista Brasileira de Fruticultura, Jaboticabal, v.29, n.1, p.172-175, 2007.

PEREIRA, J.F.M.; RASEIRA, A. Poda. In: RASEIRA, M.C.B.; PEREIRA, J.F.M.; CARVALHO, F.L.C. Pessegueiro. Brasília: Embrapa, 2014a. p.283-307.

PEREIRA, J.F.M.; RASEIRA, A. Raleio. In: RASEIRA, M.C.B.; PEREIRA, J.F.M.; CARVALHO, F.L.C. Pessegueiro. Brasília: Embrapa, 2014b. p.309-327.

PICOLOTTO, L.; MANICA-BERTO, R.; PAZIN, D.; PASA, M.S.; SCHMITZ, J.D.; PREZOTTO, M.E.; BETEMPS, D.; BIANCHI, V.J.; FACHINELLO, J.C. Características vegetativas, fenológicas e produtivas do pessegueiro cultivar Chimarrita enxertado em diferentes porta-enxertos. Pesquisa Agropecuária Brasileira, Brasília, DF, v.44, n.6, p.583-589, 2009.

RASEIRA, M.C.B.; NAKASU, B.H.; UENO, B.; SCARANARI, C. Pessegueiro: cultivar BRS Kampai. Revista Brasileira de Fruticultura, Jaboticabal, v.32, n.4, p.1275-1278, 2010.

RASEIRA, M.C.B.; NAKASU, B.H.; BARBOSA, W. Cultivares: descrição e recomendação. In: RASEIRA, M.C.B.; PEREIRA, J.F.M.; CARVALHO, F.L.C. Pessegueiro. Brasília, DF: Embrapa, 2014. p.73-141.

RAMMING, D.W.; TANNER, O. 'Nemared' peach rootstock. HortScience, Alexandria, v.18, n.3, p.376, 1983.
REIGHARD, G.L. Current directions of peach rootstock programs worldwide. Acta Horticulturae, Leuven, n.592, p.421-427, 2002.

REIGHARD, G.L.; LORETI, F. Rootstock development. In: LAYNE, D.R.; BASSI, D. The peach: botany, production and uses. Oxfordshire; CABI, 2008. p.193-220.

ROCHA, M.S.; BIANCHI, V.J.; FACHINELLO, J.C.; SCHMITZ, J.D.; PASA, M.S.; SILVA, J.B. Comportamento agronômico inicial da cv. Chimarrita enxertada em cinco porta-enxertos de pessegueiro. Revista Brasileira de Fruticultura, Jaboticabal, v.29, n.3, p.583-588, 2007.

ROSSI, C.E.; FERRAZ, L.C.C.B.; MONTALDI, P.T. Resistência de frutíferas de clima subtropical e temperado a Meloidogyne incognita raça 2 e M. javanica. Arquivos do Instituto Biológico, São Paulo, v.69, n.2, p.43-49, 2002.

SANTANA, A.S.; SANTOS, M.V.; UBERTI, A.; LUGARESI, A.; GIACOBBO, C.L.; MAYER, N.A. Genetic diversity of the genus Prunus based on per se evaluation of peach clonal rootstocks. Amazonian Journal of Agricultural and Environmental Sciences, Belém, v.63, p.1-7, 2020.

SHERMAN, W.R.; LYRENE, P.M.; SHARPE, R.H. Flordaguard peach rootstock. HortScience, Alexandria, v.26, n.4, p.427-428, 1991.

SOMAVILLA, L. Levantamento e caracterização do nematóide das galhas (Meloidogyne spp.) em Actinidia deliciosa (Chevalier) Liang \& Ferguson no Rio Grande do Sul e reação de Nicotiana tabacum $L$. e espécies frutíferas a Meloidogyne ethiopica Whitehead 1968. 2008.Dissertação (Mestrado em Agronomia) - Faculdade de Agronomia Eliseu Maciel, Universidade Federal de Pelotas, Pelotas, 2008.

WEIBEL, A.M.; REIGHARD, G.L.; RAJAPAKSE, N.C.; DEJONG, T.M. Dormant carbohydrate reserves of two peach cultivars grafted on different vigor rootstocks. Acta Horticulturae, Leuven, n.903, p.815-820, 2011. 